\title{
Experimental Validation of an ITAP Numerical Model and the Effect of Implant Stem Stiffness on Bone Strain Energy
}

\author{
K. Ahmed $0^{1},{ }^{1}$ R. J. Greene, ${ }^{2}$ W. Aston, ${ }^{3}$ T. Briggs, ${ }^{3}$ C. Pendegrass, ${ }^{1}$ M. Moazen, ${ }^{4}$ and G. Blunn ${ }^{1,5}$ \\ ${ }^{1}$ Centre for Biomedical Engineering, Institute of Orthopaedics and Musculo-Skeletal Science, University College London, \\ Stanmore HA7 4LP, UK; ${ }^{2}$ Strain Solutions Ltd., Dunston Innovation Centre, Dunston Road, Chesterfield, Derbyshire S41 \\ 8NG, UK; ${ }^{3}$ Bone Tumour Unit and Joint Reconstruction Unit, Royal National Orthopaedic Hospital, Stanmore HA7 4LP, UK; \\ ${ }^{4}$ Department of Mechanical Engineering, University College London, London WC1E 6BT, UK; and ${ }^{5}$ School of Pharmacy and \\ Biomedical Sciences, University of Portsmouth, Portsmouth PO1 2DT, UK
}

(Received 1 September 2019; accepted 10 January 2020; published online 23 January 2020)

Associate Editor Elena S. Di Martino oversaw the review of this article.

\begin{abstract}
The Intraosseous Transcutaneous Amputation Prosthesis (ITAP) offers transfemoral amputees an ambulatory method potentially reducing soft tissue complications seen with socket and stump devices. This study validated a finite element (in silico) model based on an ITAP design and investigated implant stem stiffness influence on periprosthetic femoral bone strain. Results showed good agreement in the validation of the in silico model against the in vitro results using uniaxial strain gauges and Digital Image Correlation (DIC). Using Strain Energy Density (SED) thresholds as the stimulus for adaptive bone remodelling, the validated model illustrated that: (a) bone apposition increased and resorption decreased with increasing implant stem flexibility in early stance; (b) bone apposition decreased (mean change $=-$ $9.8 \%$ ) and resorption increased (mean change $=20.3 \%$ ) from distal to proximal in most stem stiffness models in early stance. By engineering the flow of force through the implant/ bone (e.g. by changing material properties) these results demonstrate how periprosthetic bone remodelling, thus aseptic loosening, can be managed. This paper finds that future implant designs should be optimised for bone strain under a variety of relevant loading conditions using finite element models to maximise the chances of clinical success.
\end{abstract}

Keywords-Amputee biomechanics, Bone density, Bone anchored implants, Digital Image Correlation, Direct skeletal attachment, Finite Element Analysis, Osseointegration, Strain Energy Density, Strain gauge validation, Transfemoral amputees.

\footnotetext{
Address correspondence to $\mathrm{K}$. Ahmed, Centre for Biomedical Engineering, Institute of Orthopaedics and Musculo-Skeletal Science, University College London, Stanmore HA7 4LP, UK. Electronic mail: kirstin.ahmed.16@ucl.ac.uk
}

\author{
ABBREVIATIONS \\ ITAP Intraosseous Transcutaneous Amputation \\ Prosthesis \\ LC Load case \\ SAAP Skeletally Attached Amputation Prostheses
}

\section{INTRODUCTION}

Transfemoral amputees routinely ambulate using a socket (prosthetic cup) and stump (residual limb), this can lead to problems such as skin oedemas, restricted perfusion or tissue necrosis. ${ }^{33}$ Surgical alternatives offered by Skeletally Attached Amputation Prostheses (SAAP) such as the Intraosseous Transcutaneous Amputation Prosthesis (ITAP) channel load through the skeleton. This reduces the problems relating to soft tissue loading and patients cite an improved quality of life with increased prosthetic use. ${ }^{19}$

Inserting relatively stiff implants into bone results in a non-physiological distribution of load, a decrease in periprosthetic bone $\operatorname{strain}^{23}$ and culminates in bone loss and aseptic loosening. ${ }^{25,44}$ In the mechanostat mode ${ }^{16}$ the 'zone of stress equilibrium, ${ }^{35}$ proposes that a strain-related stimulus holds bone within a homeostatic range by altering the bone mass via adaptive bone remodelling (resorption or apposition). Therefore, managing the stress distribution between the implant and bone, by implant design, could manage aseptic loosening and so prevent removal or replacement surgery. 
Adaptive bone remodelling is thought to be governed by the magnitude of the bone strain, ${ }^{21}$ frequency $^{40}$ and rate of loading. ${ }^{6}$ Adaptive bone remodelling simulations using different mechanical stimulus have been compared ${ }^{34}$ and most use change in Strain Energy Density (SED) as the stimulus in both uncemented ${ }^{22}$ and cemented ${ }^{37}$ fixations.

SAAP periprosthetic bone strain measurement is not possible in vivo or in vitro due to the difficulties in obtaining measurements at the bone implant interface, however finite element (FE) models (in silico models) can generate this information. Before reliance on an in silico model can be established its accuracy must be assessed. ${ }^{1,41,42}$ Validated FE proximal femur models ${ }^{12}$ and SAAP FE models in proximal femurs ${ }^{39,44}$ are described in the literature, however at the time of writing, there is no study describing a validated in silico model of an ITAP in a proximal femur.

The aims of this work were to develop a validated FE SAAP model, based on the design of an ITAP (developed by authors) that has been used in patient clinical trials. Then to use this model to investigate the effects of SAAP implant stem stiffness on periprosthetic bone SED.

\section{MATERIALS AND METHODS}

\section{Specimen}

A human cadaveric femur from a 59 year old $86 \mathrm{~kg}$ male was sourced (Anatomy Gifts Registry, 7522 Connelley Drive Suite M, Hanover, MD 21076, USA) with similar geometry to ITAP patient number 12 in the clinical trial ${ }^{24}$ and then scanned using a Siemens Somatom Definition AS CT scanner (slice thickness = $0.6 \mathrm{~mm}$, pixel spacing $=0.35 \mathrm{~mm} \times 0.35 \mathrm{~mm}, 512 \times$ 512 matrix). The 'digital imaging and communications in medicine' images were interpolated and segmented (Scan IP, Simpleware Synopsis Inc., California, USA) to produce a $3 \mathrm{D}$ femur model from which the distal end was resected, leaving $0.201 \mathrm{~m}$ (equivalent to ITAP patient 12 residual femur length).

\section{Experimental Model (In Vitro)}

\section{The SAAP Build}

A computer aided design (CAD) model of a SAAP based on the ITAP design was generated (Solidworks, Dassault Systemes, France) and machined (Tritton tooling, Unit 21, Pages Industrial Park, LU7 4TZ, UK) from grade five titanium $\left(\mathrm{TiAl}_{6} \mathrm{~V}_{4}\right)$. The SAAP stem length was $0.12 \mathrm{~m}$ with a stem diameter distally of $12 \mathrm{~mm}$ narrowing to $9 \mathrm{~mm}$ proximally (dimensions equivalent to the ITAP of patient 12) allowing for a minimum of a $1 \mathrm{~mm}$ layer of bone cement (polymethylmethacrylate, PMMA). The collar edge shape mirrored the bone osteotomy edge (unlike the ITAP collar which was cylindrical) and the spigot was $18 \mathrm{~mm}$ in diameter, the standard size used in all ITAP patients. Four cement grooves $(1.5 \mathrm{~mm}$ deep, two radially and two longitudinally) were incorporated into the stem design as all cemented ITAP patients were of common design. No grooves were machined onto the collar surface nor was a flange added (in vivo these encourage bone ingrowth and soft tissue integration respectively), see Fig. 1.

\section{SAAP Implantation into Cadaveric Bone}

The bone was stripped of soft tissue, the femoral anteversion angle was measured before the bone's distal end was resected to leave $0.201 \mathrm{~m}$ and squared off using a calcar planer (DePuy Synthes). The fatty marrow and a small amount of cancellous bone on the endosteal surface was removed, the intramedullary (IM) canal was then washed (pulse lavage, Judd Medical, L41100) and dried. A Hardinge cement restrictor was positioned in the IM canal $10 \mathrm{~mm}$ proximal to the stem tip and a bone cement mixing system (Cemvac ${ }^{\mathrm{TM}}$, DePuy Synthes) was used to deliver the pressurised cement in a retrograde manner. At an appropriate time, the SAAP stem was inserted, and the cement allowed to set. The SAAP spigot was inserted into a stainless-steel (T303) pot and fixed with four $6 \mathrm{~mm}$ grub screws, see Fig. 1.

\section{Assembly on Load Test Bed}

The final 'assembly' (bone and SAAP) was secured to the load test bed using four M8 bolts at $6.9^{\circ}$ femoral adduction, $2.0^{\circ}$ flexion and $12.7^{\circ}$ anteversion (see assumption one). Axial load was applied through planar bearings at the femoral head on a Zwick Roell, Z005, electrodynamic testing machine (Fig. 2a).

\section{Strain Gauges}

The periosteal bone surface was cleaned, dried and smoothed with glass paper at four sites; two medially and two laterally for placement of a proximal and distal strain gauge on each. Four uniaxial gauges of $1 \mathrm{~mm}$ gauge length (Foil linear goblet gauge $1 \mathrm{~mm}$, $11^{\circ} \mathrm{C}$ STC, Tokyo Measuring Instruments Laboratory, Japan) were bonded to the bone with a flexible (1.3 GPa) adhesive (Cyanoacrylate-E, Tokyo Measuring Instruments Laboratory, Japan) along the femoral axis ( $Y$ axis in the global coordinate system), see Fig. 1. 

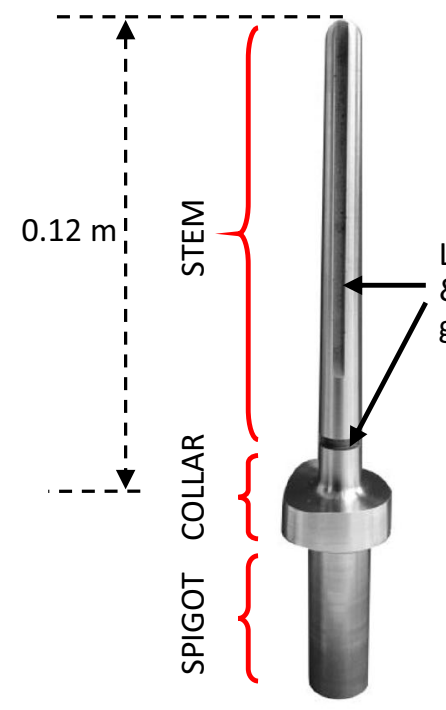

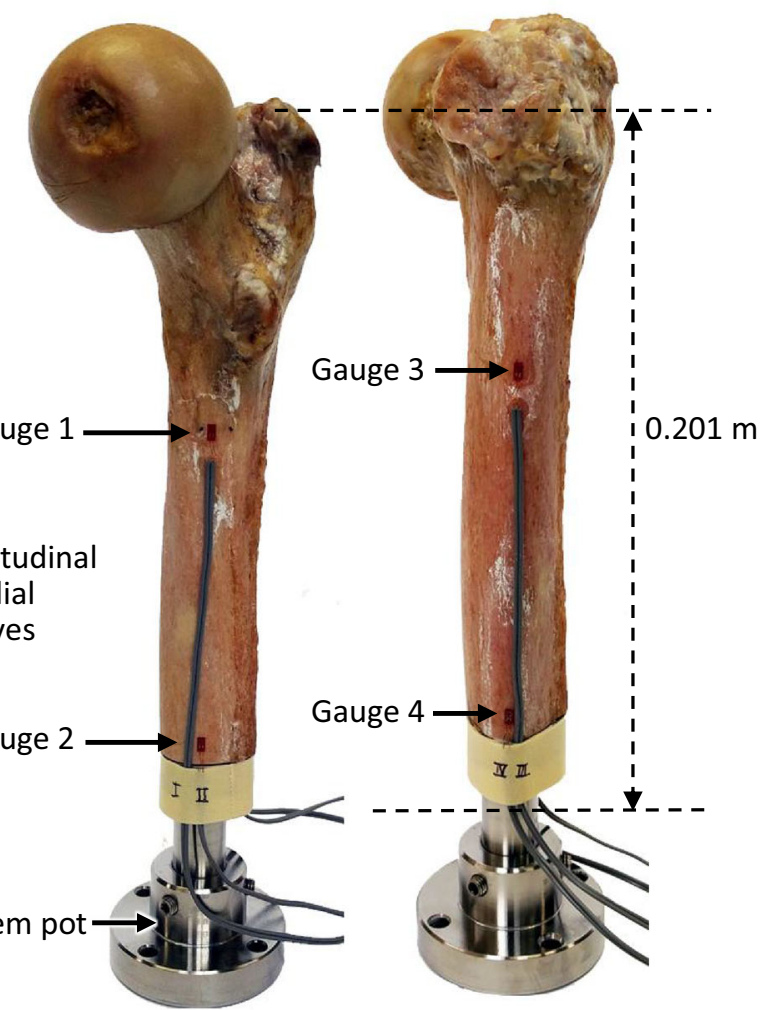

FIGURE 1. Cadaveric femur photographed medially and laterally with SAAP implanted and potted (also shown seperately). Showing locations of the strain gauges on the medial (left image) and lateral side (right image).

\section{Digital Image Correlation (DIC) Set Up}

A stereo DIC system consisting of a pair of two megapixel machine vision cameras and ruggedised fixed focal length lenses (Allied Vision Technologies Marlin F-201B, Schneider Kreuznach f1.4/17 mm). The cameras were mounted on a stiff aluminium beam, and this beam mounted on a floor standing tripod. The intrinsic/internal and extrinsic/external calibration parameters of the stereo system were determined by the simultaneous photography of a calibration target containing an array of control points, and this calibration information subsequently used to determine the triaxial location in space of each correlated image speckle subset. The calibration was conducted through a control volume which fully included the whole visible region of the bone, including distance away from the camera system. Typical uncertainty measurements of this system were of the order of one micrometre per measurement point in space.

\section{Loading}

To settle the specimen a pre-load $(100 \mathrm{~N})$ was applied, removed and the system zeroed. Incremental loads were applied as a multiple of body weight (BW) in a range consistent with data from Bergmann et al. ${ }^{3}$ in steps up (loading) and down (unloading) to account for bone's viscoelastic properties from $280.9 \mathrm{~N}(0.33 \mathrm{BW})$ to $2949.8 \mathrm{~N}$ (3.5 BW). The desired force was maintained for three seconds in which a strain measurement at each gauge and DIC stereo image pairs were recorded from the two cameras and processed using Correlated Solutions Inc. Vic3D 8 software. 
(a)

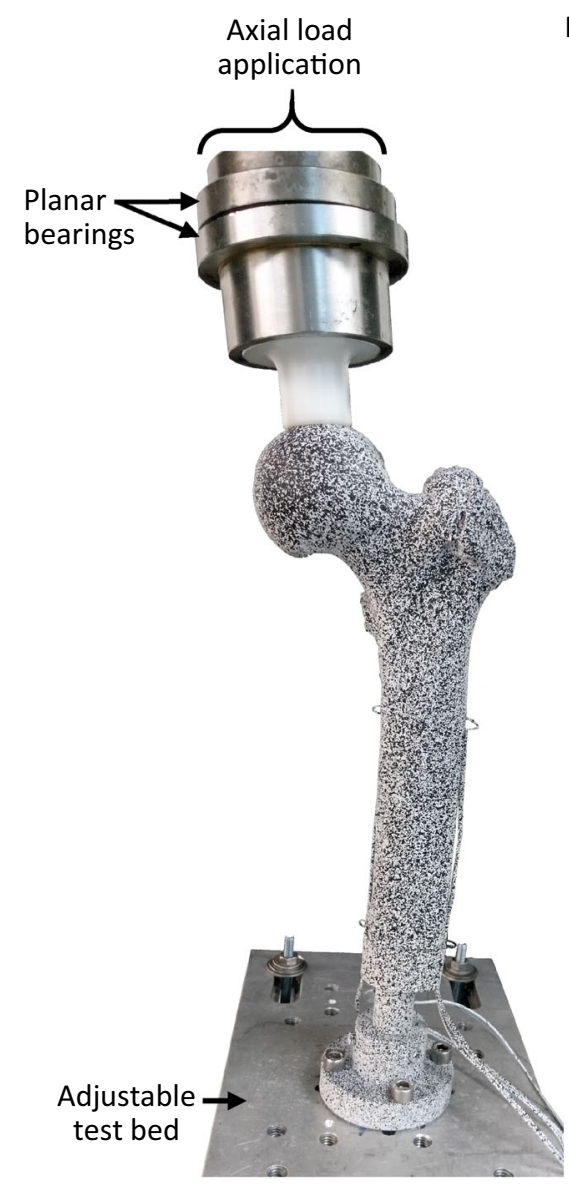

(b)

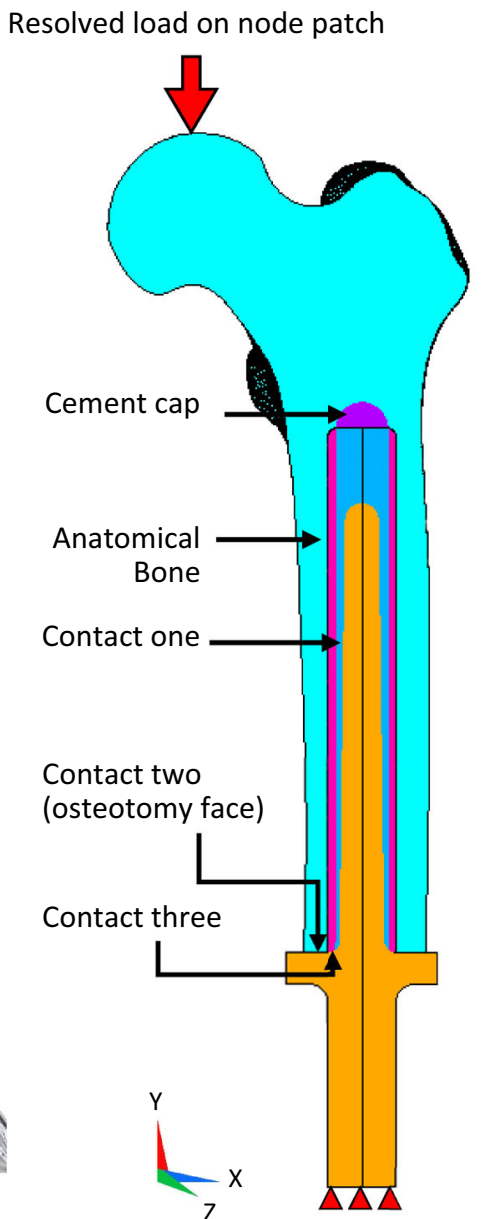

(c)

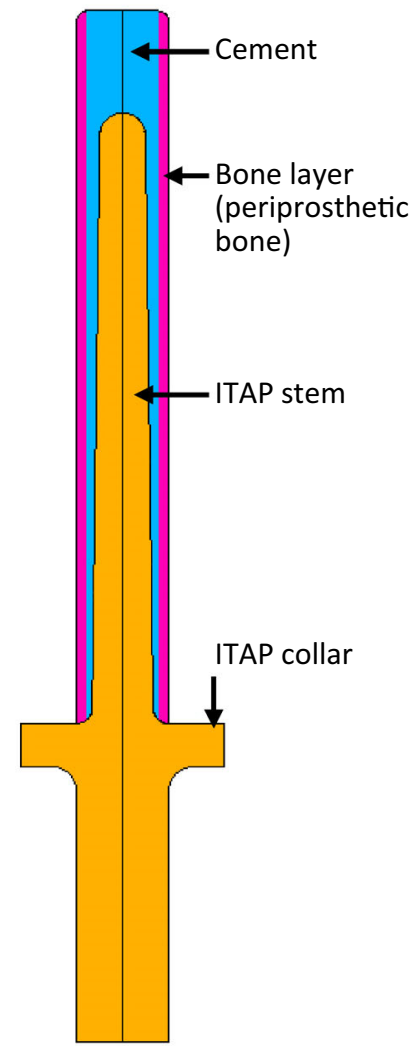

FIGURE 2. (a) In vitro model. (b) Longitudinal section of the in silico model assembly showing the bone plug inside the anatomical bone (purple cap = cement material elements, fully bonded to cement layer and anatomical bone). (c) The full bone plug.

\section{Numerical Model (In Silico)}

\section{Model Development}

The SAAP Build The dimensions of the implant were the same as those used for the in vitro work except cement grooves were not modelled and the SAAP collar was cylindrical (like the ITAP collar).

The Bone Plug Build A cylindrical bone plug was built from second order (20 noded) hexahedral elements (SOLID186) in Ansys Parametric Design Language, ANSYS (v.18.0, Ansys Inc., Pennsylvania, USA). The bone plug comprised: the SAAP, a cement layer and a bone layer (periprosthetic bone). The cement layer at the distal end was $1 \mathrm{~mm}$ thick and increased proximally, and the bone layer was uniformly $2 \mathrm{~mm}$ thick (Figs. $2 \mathrm{~b}$ and $2 \mathrm{c}$ ).
Bone Plug Insertion into Anatomical Bone Model Scan IP was used to create a cylindrical cavity within the anatomical bone model with a larger diameter than the bone's IM canal. The anatomical bone model was then positioned around the bone plug in a repeatable manner (using the image registration tool). A cement cap was fashioned to join to the top of the cement layer of the bone plug.

\section{Material Properties}

An idealised orthotropic cortical bone material model (Ashman et al..$^{2}$ ) was selected to represent the bone layer of the plug and the anatomical bone part. Properties were: $E X=12.00 \mathrm{GPa}, E Y=20.00 \mathrm{GPa}$, $E Z=13.40 \quad \mathrm{GPa}, \quad v X Y=0.22, \quad v Y Z=0.35$, $v X Z=0.38, G X Y=5.61 \mathrm{GPa}, G Y Z=6.23 \mathrm{GPa}$, 
TABLE 1. Force components in LC1 and LC2.

\begin{tabular}{llll}
\hline & FX(lateral (positive)/medial shear) & FY(proximal (positive)/distal force) & FZ(anterior (positive)/posterior shear) \\
\hline LC1 & $+101.19 \mathrm{~N}$ & $-836.19 \mathrm{~N}$ & $-29.20 \mathrm{~N}$ \\
LC2 & $-804.05 \mathrm{~N}$ & $-1957.53 \mathrm{~N}$ & $-141.95 \mathrm{~N}$ \\
\hline
\end{tabular}

$G X Z=4.53 \mathrm{GPa}$ (defined in a cylindrical coordinate system where $X=$ radial, $Z=$ circumferential, $Y=$ axial). The bone cement properties were $E=2.00$ $\mathrm{GPa}, v=0.40$ and the SAAP was modelled using the ITAP material $\left(\mathrm{TiAl}_{6} \mathrm{~V}_{4}\right) ; E=115.00 \mathrm{GPa}, v=0.30$. No cancellous bone was modelled ( $E=$ Young's modulus, $v=$ Poisson's ratio and $G=$ shear modulus).

\section{Interactions}

The cement layer was fully bonded (nodes merged) to the bone layer of the bone plug, the bone layer (second order hexahedral elements) of the bone plug was tied to the anatomical bone (second order tetrahedral elements) with multi point constraint equations, i.e. fully bonded. Three contact surfaces were modelled (Fig. 2b):

1. Between the SAAP stem and the cement layer: contact one.

2. Between the anatomical bone (osteotomy face) and the SAAP collar: contact two.

3. Between the bone layer (distal face) and the SAAP collar: contact three.

Successful ITAP surgery assumes osseointegration (fully bonded surfaces) of the distal bone and ITAP collar, however in vitro this is not the case, and the slip between the distal bone parts and the SAAP collar surface was modelled in silico by contacts two and three. The model was fully constrained distally (on the face of the SAAP spigot). All contact friction was considered isotropic with a coefficient of 0.30 .

\section{Boundary Conditions and Load Cases (LC)}

Two LC's were used, one for each part of this study (validation and effects of SAAP stem stiffness):

- LC1 (used for FE model validation): An early stance LC without muscular contribution was applied as a distributed proximal load at the femoral head with the anatomical axis of the femur colinear with the global $Y$ axis. An $842.8 \mathrm{~N}$ axial load (1.0 BW) was transformed (to account for the femoral orientation in vitro) see Table 1 . All three contacts described in the interactions section were applied to this model.

- LC2 (used with SAAP stem stiffness variations): An early stance $\mathrm{LC}$ with an intact musculoskeletal hip joint contact $\mathrm{LC}^{11}$ was similarly applied. An early stance LC was transformed using the difference between normal proximal femur $\left(10^{\circ}\right.$ flexion, $9^{\circ}$ adduction $^{7}$ ) and SAAP alignment in double legged stance (see assumption one); Table 1. Contact one only was applied; the other contact surfaces were fully bonded.

\section{Mesh Convergence}

Richardson's extrapolation ${ }^{36}$ was used to estimate the error in the solution for the bone plug model with normalised element edge lengths of $0.5,1$ and 2. A relative error of $<1 \%$ at normalised element edge length of one $(0.625 \mathrm{~mm})$ was calculated and so used (full results in appendix Table 3). Bone tetrahedral element edge lengths were matched to $0.625 \mathrm{~mm}$, total element count was 385,080 .

\section{Measurements}

Strain Gauge and DIC Node Selection Surface nodes surrounding the central node corresponding to the centre of each strain gauge in vitro, were selected and the mean axial strain was calculated for the validation.

To validate the in silico displacement, surface nodes attached to the elements representing the bone DIC visible region were selected. The nodal displacement range falling within a $95 \%$ confidence interval (to omit any outlying nodal displacements) was calculated.

SAAP Stem Stiffness The SAAP stem Young's modulus (115 GPa) was adjusted to $210 \mathrm{GPa}$ and $20 \mathrm{GPa}$, all other properties were unchanged. The stiffer stem represents biocompatible cobalt chromium $(\mathrm{CoCr}){ }^{27}$ The more flexible stem represents a cellular structured family of metals, additively manufactured from tantalum (Ta) metal. ${ }^{14}$

Strain: The SED of a solid is the work done per unit volume to deform a material from a stress free reference state to a loaded state, units are $\mathrm{Jm}^{-3}$ (or Pa). $\mathrm{SED} / \rho$ thresholds denoting a homeostatic range of $0.0036 \mathrm{Jg}^{-1} \leq$ bone mass homeostasis $\leq 0.0044 \mathrm{Jg}^{-130}$ were converted to indicate adaptive bone remodelling likelihood. Cross sections were taken at 11 equidistant $(1.09 \mathrm{~mm})$ points along the bone layer, Fig. 5a. The percentage of the area in each cross section below, 
within or above the threshold range was calculated (Adobe Photoshop CS6).

\section{Outputs}

Validation The outputs from the in vitro strain gauges were compared to in silico strain in the longitudinal global $(Y)$ axis and agreement was measured using the bivariate analysis, Lin's Concordance Correlation Coefficient (CCC). ${ }^{28}$ The in vitro DIC displacement maps were compared (a.) visually and (b.) as a span of displacement $(\mathrm{mm})$ to the corresponding field of view in silico, agreement was quantified using CCC.

Implant Stem Stiffness SED results from the in silico analysis were computed at each of the 11 cross sections of bone layer in each of the three stem stiffness models. SED in regions below or above the thresholds were considered likely to experience adaptive bone remodelling (resorption or apposition respectively).

\section{Sensitivity Analysis}

Sensitivity of axial periosteal bone strain at the four gauge sites was investigated in parameters likely to influence a static structural FE analysis. These were bone material and contact properties between parts. A total of 65 models were run.

\section{Assumptions}

\section{Assumption one}

The assumption that the orientation of the SAAP patient's femur in early stance being similar to double leg stance has been made in this study. In the absence of joint angle data in the literature for SAAP patients, observations by prosthetists from fluoroscopy results at the $\mathrm{RNOH}$ in double leg stance were used.

\section{Assumption two}

This study assumes that local SED values provide an indication to the bone's likely initial response to ITAP implantation (local resorption, maintenance or apposition).

\section{RESULTS}

\section{Sensitivity Analysis}

Results were normalised by calculating one standard deviation (SD) as a percentage of the mean strain at each gauge of each model pertaining to the parameter of interest.
- Axial bone strain was sensitive to material property changes in non-linear (contact) models. Gauges one, two and three resulted in sensitivities $<15 \%$, gauge four was $21 \%$.

- Contact types ('standard', 'no separation', 'bonded', 'rough' as defined in the ANSYS contact technology manual) had a profound effect on gauges two and four $(23 \%$ and $88 \%$ respectively), but less in gauges one and three ( $1 \%$ and $0.3 \%$ respectively).

- The effect of modelling the osteotomy contact surface as $50 \%$ bonded resulted in sensitivities $<8 \%$ in all gauges apart from gauge four which was $20 \%$.

- Axial bone strain was relatively insensitive to changes in spring stiffness coefficients in rotation and translation between the ITAP spigot and the stem pot (modelling in vitro micromotion in the fixing) with sensitivity in all gauges $<10 \%$.

- Axial bone strain sensitivity in the friction models was low $(<5 \%)$ in all gauges except gauge four which was $23 \%$.

\section{Validation}

\section{Strain Gauge Validation}

The CCC produced a correlation $\rho_{\mathrm{c}}=0.934$ between the four mean in silico and in vitro strain gauge results, Fig. 3. In silico strains corresponding to gauge positions one, three and four (error $=12.17 \%$, $10.62 \%$ and $9.58 \%$ respectively) were closer to their corresponding mean in in vitro strains than gauge two (error $=30.79 \%)$, Table 2 .

\section{DIC Validation}

Investigating the span of displacement in vitro and in silico, generated acceptable agreement (Table 2: error $=3.27 \%, 5.85 \%$ and $11.79 \%$ for displacement in $X, Y$ and $Z$ respectively) with a CCC of 0.997 , Fig. 3 .

Figure 4 illustrates the full field displacement data in vitro and in silico:

$Y$ axis: $\quad$ Displacement along the $Y$-axis was maximum (positive) along the lateral edge and maximum (negative) along the medial edge of the bone DIC record in silico and in vitro.

$X$ axis: $\quad$ The largest displacements in silico and in vitro along the $X$-axis were proximal and decreased distally.

$Z$ axis: $\quad$ Along the $Z$-axis, maximum (negative) displacement was recorded at the greater trochanter in vitro and in silico and decreased in a diagonal fashion to a minimum at the femoral head in vitro and in silico. 
STRAIN GAUGING: Concordance coefficient, $\rho_{c}=0.934$

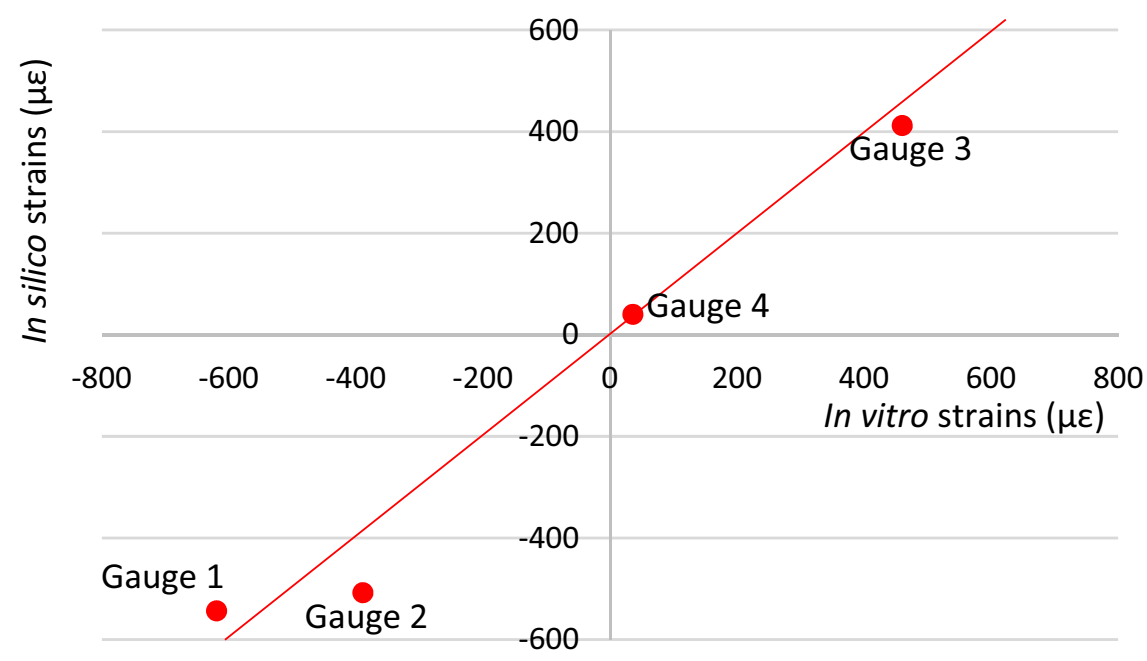

DIC: Concordance coefficient, $\rho_{c}=0.997$

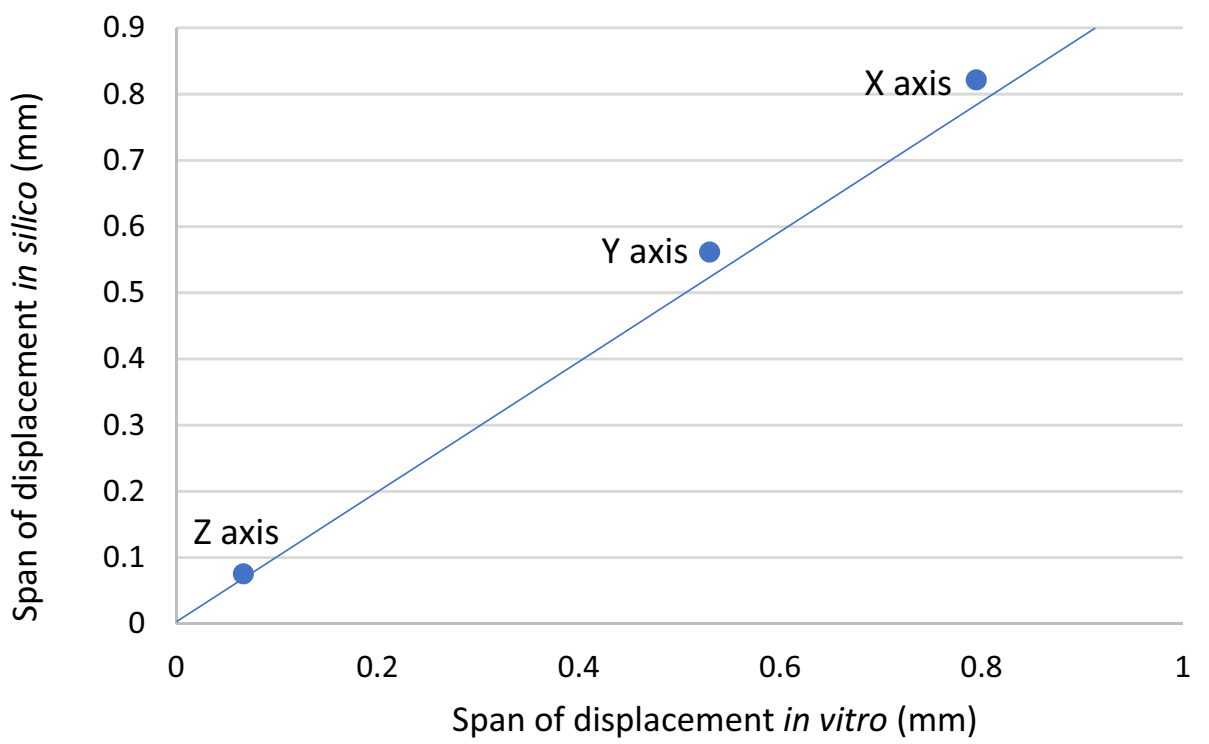

FIGURE 3. Top $=$ plot in vitro against in silico strain $(\mu \varepsilon)$. Bottom $=$ plot in vitro against in silico displacement $(\mathrm{mm})$.

TABLE 2. Top $=$ Mean strain $(\mu \varepsilon)$ in vitro and in silico with SD in brackets under LC1. Bottom $=$ displacement $(\mathrm{mm})$ in vitro and in silico at all gauges/axes under LC1.

\begin{tabular}{lllll}
\hline Strain $(\mu \varepsilon)$ & \multicolumn{1}{c}{ Gauge 1 } & Gauge 2 & Gauge 3 & Gauge 4 \\
\hline Mean in vitro & $-619.0(5.2)$ & $-388.5(8.5)$ & $460.5(2.9)$ & $36.5(12.7)$ \\
Mean in silico & -543.65 & -508.12 & 411.58 & 39.997 \\
Error $(\%)$ & 12.17 & 30.79 & 10.62 & 9.58 \\
\hline Displacement $(\mathrm{mm})$ & $X$ axis & Y axis & Z axis \\
\hline In vitro span & 0.795 & 0.53 & 0.067 \\
In silico span & 0.821 & 0.561 & 0.0749 \\
Error $(\%)$ & 3.27 & 5.85 & 11.79 \\
\hline
\end{tabular}




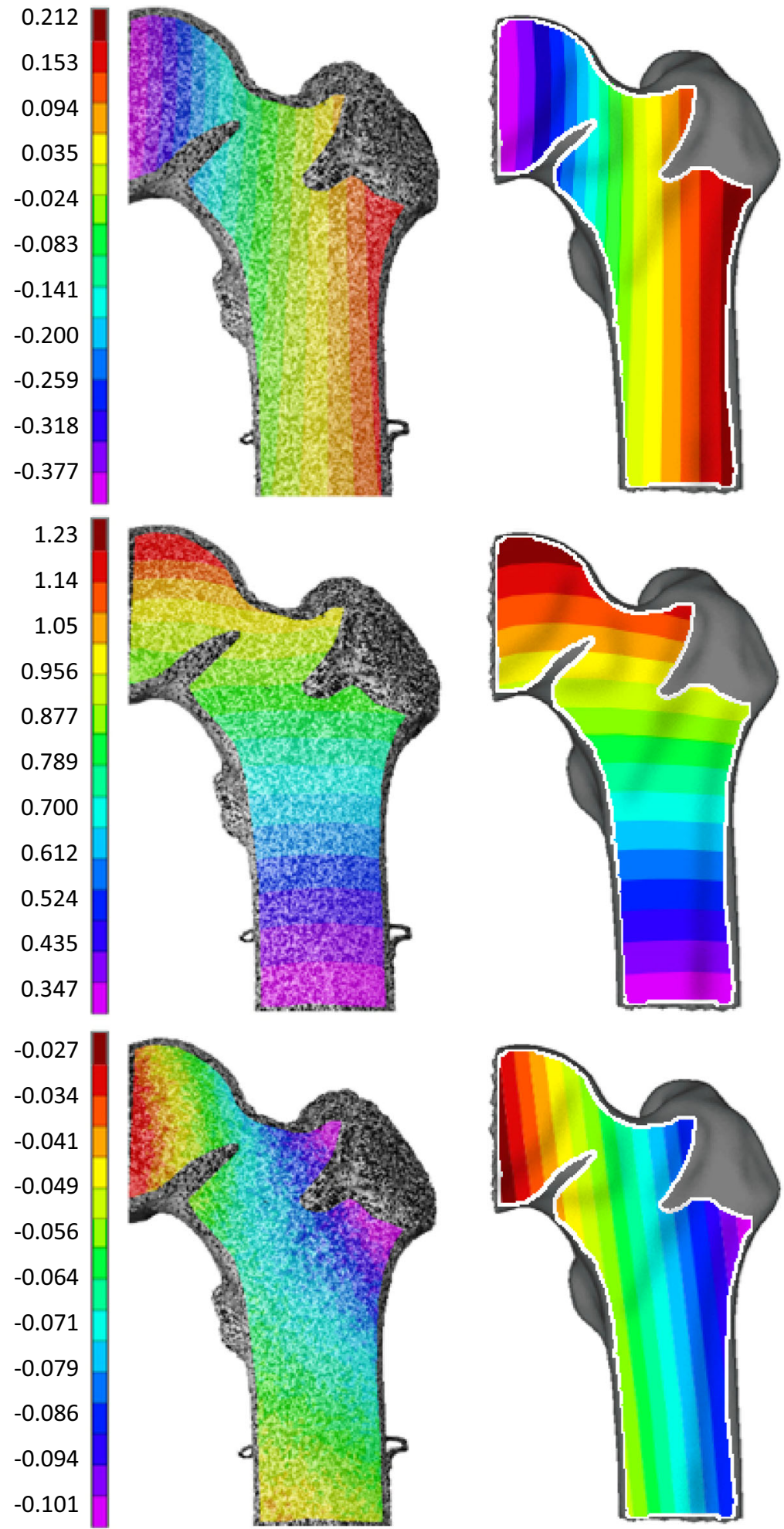

FIGURE 4. In vitro displacement $(\mathrm{mm})$ on the left, in silico displacement $(\mathrm{mm})$ on the right. The white line on the in silico plots bounds the equivalent DIC camera view area. Top $=Y$ axis, middle $=X$ axis, bottom $=Z$ axis. 


\section{SAAP Stem Material Change}

Figure 5a shows the bone assembly without the SAAP with the location of the 11 periprosthetic bone cross sections (slices). An LC2 load resulted in a mediolateral bending moment about the $Z$ axis decreasing moving proximally (Fig. $5 \mathrm{~b}$ showing the two bone parts only in transverse section).

Figure 5c illustrates the effect on the periprosthetic bone of increasing SAAP stem stiffness (from left to right) under LC2; a reduction in SED medially and laterally is observed. The maximum SED in the more flexible stems in the periosteal bone were $14 \%$ and $27 \%$ higher when comparing $20 \mathrm{GPa}$ vs. $115 \mathrm{GPa}$ and $115 \mathrm{GPa}$ vs. $210 \mathrm{GPa}$ models respectively. On the periprosthetic bone there was a $50 \%$ increase when comparing $20 \mathrm{GPa}$ vs. $115 \mathrm{GPa}$ stems and a $13 \%$ increase when comparing $115 \mathrm{GPa}$ vs. $210 \mathrm{GPa}$.

Percentage of total slice area above and below the SED threshold (indicating apposition and resorption respectively) are plotted for slices 1-10 in all stem stiffness models in Fig. 6. Using SED thresholds as the signal for adaptive bone remodelling this shows that (a.) there is more periprosthetic bone apposition in the more flexible stemmed models and (b.) that periprosthetic bone apposition decreases in all stem stiffness models moving proximally. (c.) There is less periprosthetic bone resorption in the more flexible stemmed models and (d.) that periprosthetic bone resorption increases in most stem stiffness models moving proximally. There is an anomaly proximal to slice seven in the $20 \mathrm{GPa}$ stemmed model as resorption area decreases up to slice ten.

\section{DISCUSSION}

\section{Sensitivity Analysis}

The large degree to which bone stiffness and stiffness orientation influenced axial strain results is due to their effect on bone tissue deformation. Most transfemoral amputees present with osteopenic bone through disuse, ${ }^{17}$ the decrease in bone mineral density $(\rho)$ is related to Young's modulus $(E)$ by the power law $E=a \rho^{\mathrm{b}}$ where $a$ and $b$ are constants. ${ }^{20}$ Osteopenia inclusion is therefore critical for accurate FE models of SAAP patient assemblies. Cancellous bone was omitted from the model in this study as there was no discernible difference in the axial strain results at any of the gauge sites. This was not unexpected since the most significant effect of LC1 and LC2 loading was to produce a mediolateral bending moment about the $Z$ axis in the diaphysis. Due to the bone plug occupying the entire intramedullary canal, the only cancellous bone that was omitted was that in the femoral head. Distal gauge site axial bone strains were highly sensitive to the type and number of contact surfaces employed under LC1 or LC2 suggesting some conflicting convergence criteria. Although three contact surfaces best models the effect of slip in vitro at the osteotomy face, caution should be exercised making this choice due to the large error observed in vitro axial strain in gauges 2 and 4 .

\section{Validation}

A robust discrete point validation corroborated by the full field validation of the FE model has been presented however there were some notable potential sources of validation discrepancy: There appeared to be conflicting convergence criteria when 3 distal contacts were modelled in silico; the increased accuracy of the proximally located gauges echoes this finding. Furthermore, discrepancies could have been introduced by visual placement of the uniaxial gauge on the bone being subject to misalignment with respect to the $Y$ axis. Additionally, greyscale data from the cadaveric bone CT scan did provide inhomogeneous bone material properties (using density modulus relationships), however these were not employed in either bone part in the in silico model. Since the bone plug was housed inside the anatomical bone, both the interface between the outer surface of the plug and the anatomical bone as well as the elements within the anatomical bone would have experienced a step change in elastic modulus. This could have led to a disturbance in the stress distribution between these regions, ${ }^{29}$ potentially resulting in spurious behaviour and so an idealised homogenous cortical bone material (for both bone parts) was selected instead. Lastly, generation of strain information requires local differentiation of the displacement information and inevitably suffers from the introduction of noise and artefacts from the strain calculation algorithm.

Use of single-grid uniaxial strain gauge coupons is an effective method of recording the in vitro strain in one direction. It also avoids the use of stacked rosettes where three gauge grids are superposed onto the same measurement location which results in a thick gauge coupon, is difficult to adhere to a curved bone surface and may affect the strain readings. Acceptable in silico agreement was observed with a CCC of 0.934; discrete point gauge discrepancies and correlations of this order are similar to those of comparable biomechanical studies. ${ }^{5,31}$ 


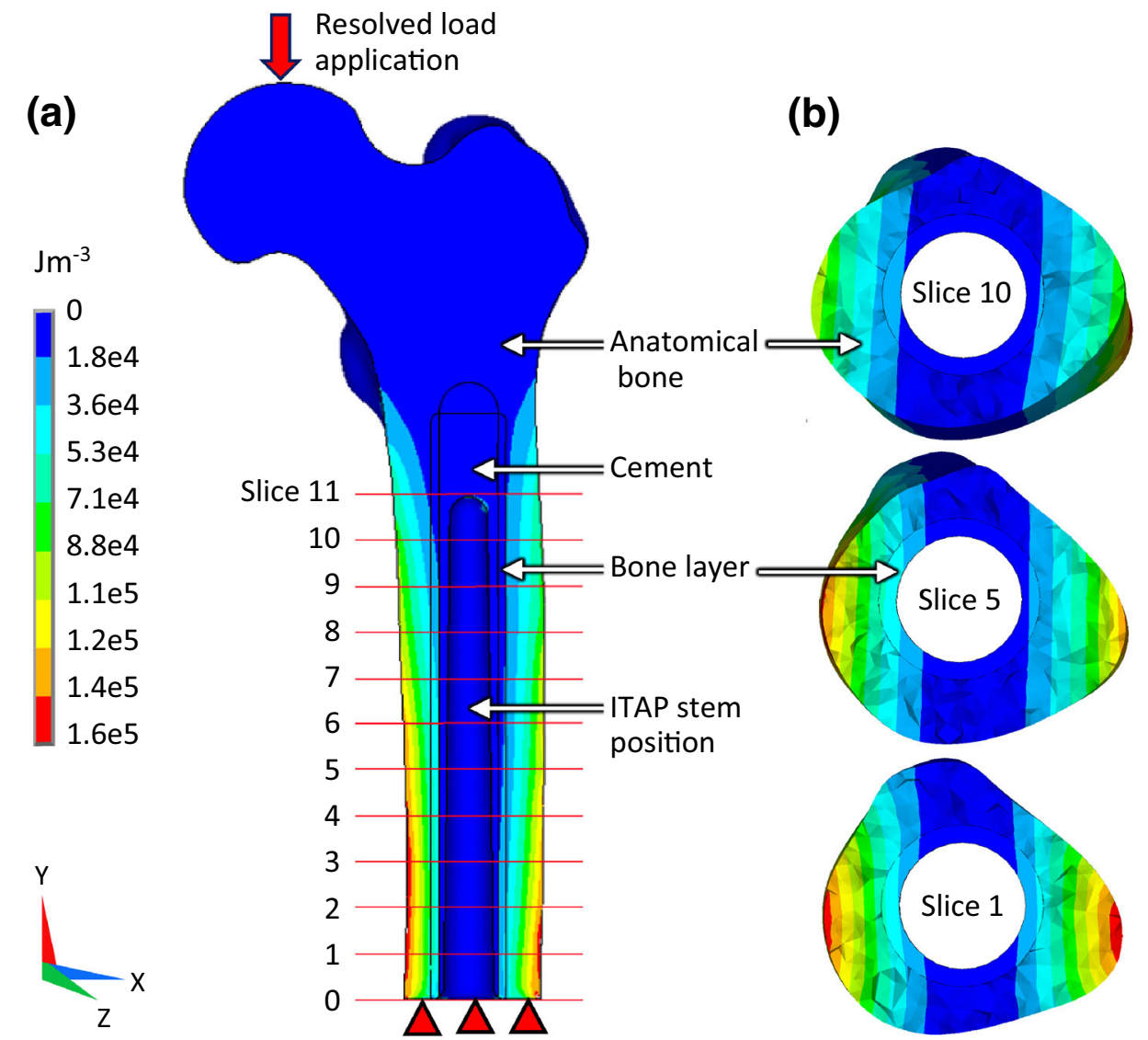

(c)
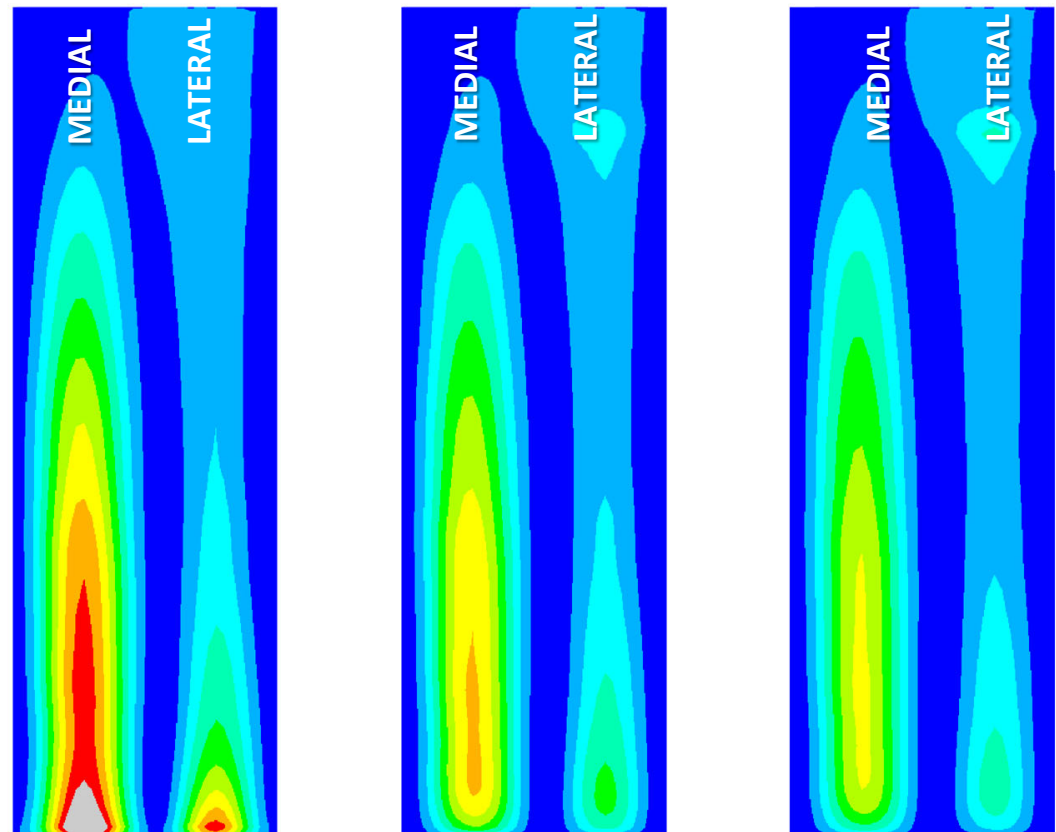

FIGURE 5. (a) SED $\left(\mathrm{Jm}^{-3}\right)$ in a longitudinal section of the assembly (minus ITAP) showing slice positions 0-11 at 1.09 mm intervals in the periprosthetic bone under LC2 with a $115 \mathrm{GPa}$ stem. (b) SED in transverse section of the bone (anatomical bone + bone layer) at slice locations 1, 5 and 10 under LC2 with a $115 \mathrm{GPa}$ stem. (c) Inner surface of periprosthetic bone layer 'unwrapped' showing SED contours in models with a $20 \mathrm{GPa}$ (left), $115 \mathrm{GPa}$ (middle) and $210 \mathrm{GPa}$ (right) stiffness stem. 

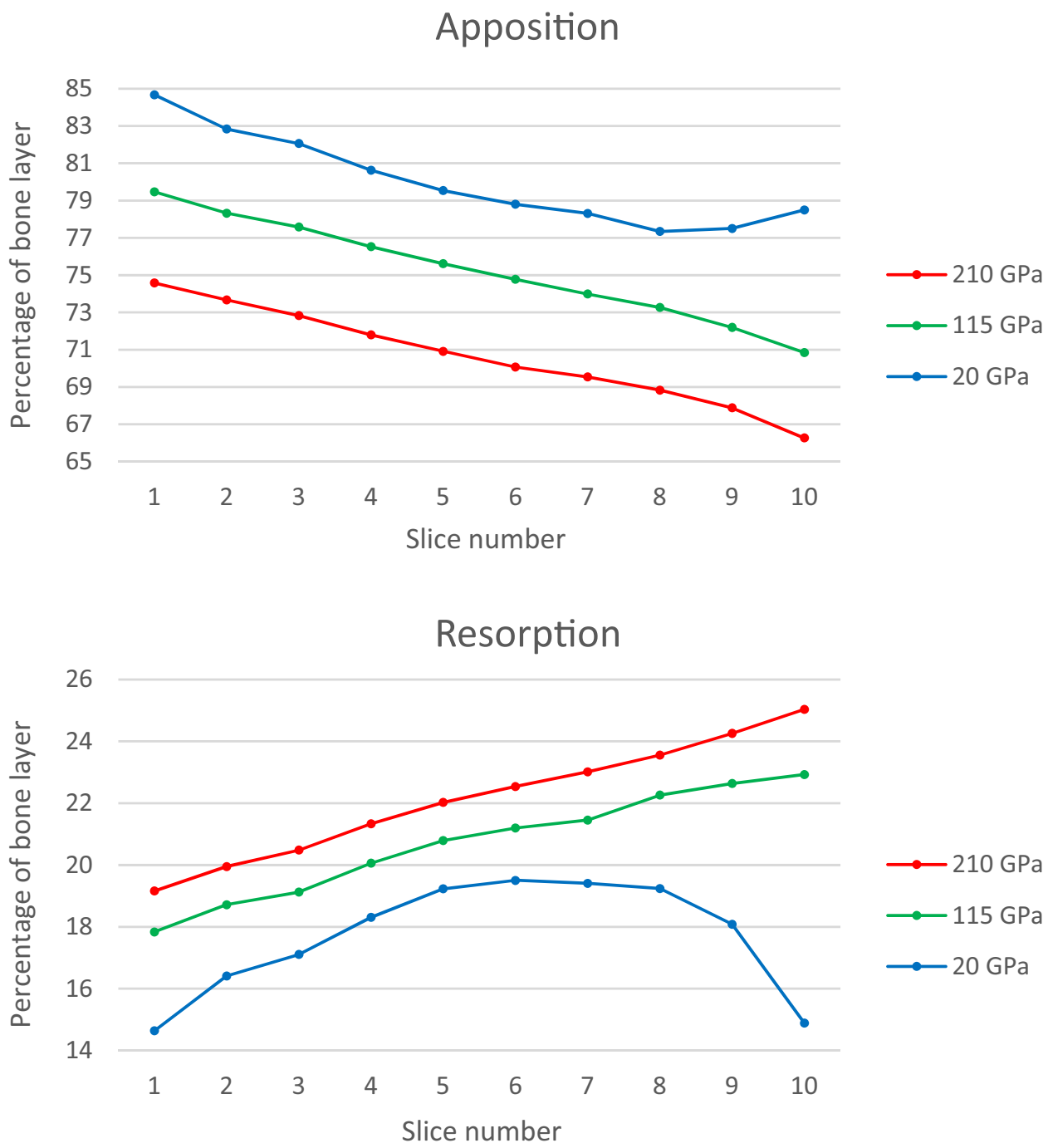

FIGURE 6. Bone remodelling with respect to SED thresholds along the bone layer (periprosthetic bone) from the first layer proximal to the osteotomy face (slice 1) to the last layer distal to the tip of the ITAP (slice 10) each $1.09 \mathrm{~mm}$ apart.

\section{DIC Validation}

Displacement information from the DIC method is of attractive precision and high signal to noise ratio. Since the full surface displacement fields are available from the in silico model presented here, a direct comparison has been made between displacement fields, thus avoiding the difficulties associated with the calculation of the second order strain data from the first order displacement information. The displacement field span demonstrates good agreement with slightly larger displacements in silico in all axis compared to in vitro, with an average error of $7 \%$ and a CCC of 0.997. It is possible that the discrepancy between the in vitro and in silico displacements in the $Z$ axis are the result of a torsion that was not calculated by the in silico model. A possible reason for this may have been the way that the force was applied or accuracy of the measured angle of anteversion, none the less discrepancies of this magnitude are not unexpected in comparable DIC biomechanical studies. ${ }^{12,18}$ Comparison between the experimentally derived displacements and those predicted by simulation would be further improved by the introduction of discrete points of comparison between the two data fields - this will be the subject of future work, with additional full-field mapping of the DIC and FE results.

\section{Implant Stem Material, SED and Bone Remodelling}

Managing aseptic loosening of SAAP due to periprosthetic bone resorption is key to clinical success, as studies using similar endoprostheses have 
shown. ${ }^{4,8,9}$ Endosteal resorption will destabilise the implant, conversely if osseointegration and bone growth into the collar can be achieved without radiolucency, then the implant will be stabilised. ${ }^{8,15}$ The damage repair theory suggests that when damage from fatigue or impact occur, bone can detect, remove and replace it within resorption cavities. ${ }^{32}$ Immediately post surgically and over time, impact and fatigue damage signals (such as microcracks cutting through the processes of osteocytes ${ }^{13}$ and/or osteocyte apoptosis) will affect the remodelling output as well as the SED remodelling signal. In silico models in this study have shown periprosthetic adaptive bone remodelling changes in response to SAAP stem stiffness modification (Figs. 5c and 6).

Since each part of the assembly will carry a portion of the load proportional to its stiffness results were as expected; a higher SED in periprosthetic bone when the stem stiffness was reduced (therefore a larger area of the bone crossed the SED apposition threshold) and vice versa. Furthermore, the distribution of strain energy was greatest distally and decreased proximally (Figs. 5a, 5b, 5c and 6). Summation of the bending moments (Varignon's theorem) produced from the components of LC2 will deliver this approximate solution.

FX of LC1 is positive whereas in LC2 it becomes negative as the adductor muscles generate the medial forces of early stance. ${ }^{26}$ The value of patient specific load cases, bone models and implant design in predicting regions of adaptive remodelling will be critical for accurate FE modelling of SAAP patient assemblies. To date this has not been a consideration for transfemoral implants but may be important in the design of individualised implants and in the positioning of the external prostheses relative to the spigot.

Obtaining similar strain results to this study in a collared SAAP design, Tomaszewski et al. ${ }^{38}$ demonstrated the effect of stem material change on periosteal bone strain. Using experimental and numerical models they showed that the distal and middle gauges and nodes respectively, in three different loading cases, experienced strains $21-29 \%$ higher using a more flexible stem. In other SAAP designs with a stiff stem (115 $\mathrm{GPa}$ ), such as the Osseointegrated Prostheses for the
Rehabilitation of Amputees (screw fit), distal bone resorption has been shown clinically and in numerical models. ${ }^{44}$ The inclusion of a SAAP collar in pressfit designs such as the ITAP appears instrumental in managing distal bone strain, hence clinical success.

Manufacture of porous metals is by electron or laser beam sintering a metal powder; the resultant material fatigue limit is usually exceeded due to the nucleation of cracks from pores. ${ }^{45}$ In the case of a fully porous load bearing SAAP stem, especially one that may not be ingrown by bone (this cannot be assumed), further work needs to be undertaken to ascertain the risk of implant fracture. Hypothetically, a porous stem blended into a solid collar and spigot would be the design goal.

In transfemoral amputees muscle groups are removed or transacted and only partly functioning which contributes to osteopenia and remodelling. ${ }^{17}$ Periosteal and endosteal bone resorption will decrease the cortical area and the bone's resistance to bending and in combination with a decrease in bone density, presents a different material to a stress analysis than the one used in this study. Accordingly, adaptive bone remodelling may produce a different material distribution and a transient analysis using a bone remodelling algorithm ${ }^{10,43}$ may be a consideration to monitor the bone change over time.

Using SED as the key indicator for periprosthetic adaptive bone remodelling the value of implant stiffness has been demonstrated. This validated numerical model will allow further studies to be conducted in order to quantify bone remodeling considering variations such as implant material, geometry and fixation type. These encouraging results could mean that future SAAP implant designs should be optimised for bone strain under a variety of relevant loading conditions using FE models to maximise the chances of clinical success.

\section{APPENDIX}

See Table 3. 
TABLE 3. Results of Richardson's extrapolation for bone plug with a constant grid refinement ratio $\left(r=h_{3} / h_{2}=h_{2} / h_{1}=\right.$ constant $)$ and the observed convergence rate obeying: $p=\frac{\log \left(\frac{f_{3}-f_{2}}{t_{2}-f_{1}}\right)}{\log r}$ such that f.exact $\approx f_{1}-\frac{f_{2}-f_{1}}{r_{21}^{p}-1}$

\begin{tabular}{lll}
\hline & Most coarse mesh & \\
\hline Normalised element edge length, $h$ & 2 & 1 \\
Maximum stress in Y axis (Pa), $f$ & $1,421,900$ & $1,422,700$ \\
Element edge length refinement ratio, $r$ & & 2.000 \\
Relative error, $e$ & & $0.056 \%$ \\
Error to exact solution & & $0.008 \%$ \\
Grid Convergence Index, GCl & & $0.000 \%$ \\
95\% Confidence interval & & $0.001 \%$ \\
$\quad$ Lower bound & & $0.001 \%$ \\
$\quad$ Upper bound & & $1,422,800$ \\
Estimate of exact solution, f.exact & $1,422,782.143$ \\
\hline
\end{tabular}

\section{ACKNOWLEDGMENTS}

This study was significantly enriched by Angus Ramsay of Ramsay Maunder Associates Ltd., who is a specialist in the ANSYS software. No benefits in any form have been or will be received from a commercial party related directly or indirectly to the subject of this manuscript.

\section{OPEN ACCESS}

This article is licensed under a Creative Commons Attribution 4.0 International License, which permits use, sharing, adaptation, distribution and reproduction in any medium or format, as long as you give appropriate credit to the original author(s) and the source, provide a link to the Creative Commons licence, and indicate if changes were made. The images or other third party material in this article are included in the article's Creative Commons licence, unless indicated otherwise in a credit line to the material. If material is not included in the article's Creative Commons licence and your intended use is not permitted by statutory regulation or exceeds the permitted use, you will need to obtain permission directly from the copyright holder. To view a copy of this licence, visit http://crea tivecommons.org/licenses/by/4.0/.

\section{REFERENCES}

\footnotetext{
${ }^{1}$ Anderson, A. E., B. J. Ellis, and J. A. Weiss. Verification, validation and sensitivity studies in computational biomechanics. Comput. Methods Biomech. Biomed. Eng. 10:171184, 2007

${ }^{2}$ Ashman, R. B., S. C. Cowin, W. C. Van Buskirk, and J. C. Rice. A continuous wave technique for the measurement of the elastic properties of cortical bone. J. Biomech. 17:349361,1984
}

${ }^{3}$ Bergmann, G., A. Bender, J. Dymke, G. Duda, and P. Damm. Standardized loads acting in hip implants. PLoS ONE 11:e0155612, 2016.

${ }^{4}$ Blunn, G. W., and M. E. Wait. Remodelling of bone around intramedullary stems in growing patients. J. Orthop. Res. 9:809-819, 1991.

${ }^{5}$ Bougherara, H., R. Zdero, S. Shah, M. Miric, M. Papini, P. Zalzal, and E. H. Schemitsch. A biomechanical assessment of modular and monoblock revision hip implants using FE analysis and strain gage measurements. J. Orthop. Surg. Res. 5:34, 2010.

${ }^{6}$ Burr, D. B., A. G. Robling, and C. H. Turner. Effects of biomechanical stress on bones in animals. Bone 30:781786, 2002.

${ }^{7}$ Cherian, J. J., B. H. Kapadia, S. Banerjee, J. J. Jauregui, K. Issa, and M. A. Mont. Mechanical, anatomical, and kinematic axis in TKA: concepts and practical applications. Curr. Rev. Musculoskelet. Med. 7:89-95, 2014.

${ }^{8}$ Coathup, M. J., V. Batta, R. C. Pollock, W. J. Aston, S. R. Cannon, J. A. Skinner, T. W. Briggs, P. S. Unwin, and G. W. Blunn. Long-term survival of cemented distal femoral endoprostheses with a hydroxyapatite-coated collar: a histological study and a radiographic follow-up. J. Bone Jt. Surg. Am. 95:1569-1575, 2013.

${ }^{9}$ Coathup, M. J., A. Sanghrajka, W. J. Aston, P. D. Gikas, R. C. Pollock, S. R. Cannon, J. A. Skinner, T. W. R. Briggs, and G. W. Blunn. Hydroxyapatite-coated collars reduce radiolucent line progression in cemented distal femoral bone tumor implants. Clin. Orthop. Relat. Res. 473:1505-1514, 2015

${ }^{10}$ Cowin, S. C., and D. H. Hegedus. Bone remodeling I: theory of adaptive elasticity. J Elasticity 6:313-326, 1976.

${ }^{11}$ Database « OrthoLoadat https://orthoload.com/database/.

${ }^{12}$ Dickinson, A. S., A. C. Taylor, H. Ozturk, and M. Browne. Experimental validation of a finite element model of the proximal femur using digital image correlation and a composite bone model. J. Biomech. Eng. 133:014504, 2011.

${ }^{13}$ Dooley, C., P. Tisbo, T. C. Lee, and D. Taylor. Rupture of osteocyte processes across microcracks: the effect of crack length and stress. Biomech. Modeling Mechanobiol. 11:759766, 2012.

${ }^{14}$ Eid, Y. The myths of trabecular metal: 'the next best thing to bone'. Egypt Orthop. J. 48:327, 2013.

${ }^{15}$ Fromme, P., G. W. Blunn, W. J. Aston, T. Abdoola, J. Koris, and M. J. Coathup. The effect of bone growth onto massive prostheses collars in protecting the implant from fracture. Med. Eng. Phys. 41:19-25, 2017. 
${ }^{16}$ Frost, H. M. Bone mass and the mechanostat: a proposal. Anat. Rec. 219:1-9, 1987.

${ }^{17}$ Gailey, R. Review of secondary physical conditions associated with lower-limb amputation and long-term prosthesis use. JRRD 45:15-30, 2008.

${ }^{18}$ Grassi, L., S. P. Väänänen, S. Amin Yavari, H. Weinans, J. S. Jurvelin, A. A. Zadpoor, and H. Isaksson. Experimental validation of finite element model for proximal composite femur using optical measurements. J. Mech. Behav. Biomed. Mater. 21:86-94, 2013.

${ }^{19}$ Hagberg, K., E. Hansson, and R. Brånemark. Outcome of percutaneous osseointegrated prostheses for patients with unilateral transfemoral amputation at two-year follow-up. Arch. Phys. Med. Rehabil. 95:2120-2127, 2014.

${ }^{20}$ Helgason, B., E. Perilli, E. Schileo, F. Taddei, S. Brynjólfsson, and M. Viceconti. Mathematical relationships between bone density and mechanical properties: a literature review. Clin. Biomech. 23:135-146, 2008.

${ }^{21}$ Huiskes, R., R. Ruimerman, G. H. van Lenthe, and J. D. Janssen. Effects of mechanical forces on maintenance and adaptation of form in trabecular bone. Nature 405:704 706, 2000.

${ }^{22}$ Huiskes, R., H. Weinans, H. J. Grootenboer, M. Dalstra, B. Fudala, and T. J. Slooff. Adaptive bone-remodeling theory applied to prosthetic-design analysis. J. Biomech. 20:1135-1150, 1987.

${ }^{23}$ Huiskes, R., H. Weinans, and B. V. Rietbergen. The relationship between stress shielding and bone resorption around total hip stems and the effects of flexible materials. Clin. Orthop. Relat. Res. NA;124-134, 1992.

${ }^{24}$ Intraosseous Transcutaneous Amputation Prosthesis-Full Text View-ClinicalTrials.govat https://clinicaltrials.gov/c t2/show/NCT02491424.

${ }^{25}$ Jeyapalina, S., J. P. Beck, R. D. Bloebaum, and K. N. Bachus. Progression of bone ingrowth and attachment strength for stability of percutaneous osseointegrated prostheses. Clin. Orthop. Relat. Res. 472:2957-2965, 2014.

${ }^{26}$ John, C. T., A. Seth, M. H. Schwartz, and S. L. Delp. Contributions of muscles to mediolateral ground reaction force over a range of walking speeds. J. Biomech. 45:24382443, 2012.

${ }^{27}$ Learmonth, I. D., C. Young, and C. Rorabeck. The operation of the century: total hip replacement. Lancet 370:1508-1519, 2007.

${ }^{28}$ Lin, L., and L. D. Torbeck. Coefficient of accuracy and concordance correlation coefficient: new statistics for methods comparison. PDA J. Pharm. Sci. Technol. 52:5559, 1998

${ }^{29}$ Maunder, E. A. W., J. P. M. D. Almeida, and A. C. A. Ramsay. A general formulation of equilibrium macro-elements with control of spurious kinematic modes: the exorcism of an old curse. Int. J. Num. Methods Eng. 39:3175-3194, 1996.

${ }^{30}$ Mellal, A., H. W. A. Wiskott, J. Botsis, S. S. Scherrer, and U. C. Belser. Stimulating effect of implant loading on surrounding bone. Clin. Oral Implants Res. 15:239-248, 2004.

${ }^{31}$ Moazen, M., J. H. Mak, L. W. Etchels, Z. Jin, R. K. Wilcox, A. C. Jones, and E. Tsiridis. The effect of fracture stability on the performance of locking plate fixation in periprosthetic femoral fractures. J. Arthroplast. 28:15891595, 2013.

${ }^{32}$ Parfitt, A. M. Osteonal and hemi-osteonal remodeling: the spatial and temporal framework for signal traffic in adult human bone. J. Cell. Biochem. 55:273-286, 1994.

${ }^{33}$ Pascale, B. A., and B. K. Potter. Residual limb complications and management strategies. Curr. Phys. Med. Rehabil. Rep. 2:241-249, 2014.

${ }^{34}$ Piccinini, M., J. Cugnoni, J. Botsis, P. Ammann, and A. Wiskott. Numerical prediction of peri-implant bone adaptation: comparison of mechanical stimuli and sensitivity to modeling parameters. Med. Eng. Phys. 38:1348-1359, 2016.

${ }^{35}$ Prendergast, P. J., and D. Taylor. Prediction of bone adaptation using damage accumulation. J. Biomech. 27:1067-1076, 1994.

${ }^{36}$ Richardson, L. F., and J. A. Gaunt. The deferred approach to the limit. Part I. Single lattice. Part II. Interpenetrating lattices. Philos. Trans. R. Soc. A 226:299-361, 1927.

${ }^{37}$ Sanjay, D., S. Mondal, R. Bhutani, and R. Ghosh. The effect of cement mantle thickness on strain energy density distribution and prediction of bone density changes around cemented acetabular component. Proc. Inst. Mech. Eng. H 232:912-921, 2018.

${ }^{38}$ Tomaszewski, P. K., B. Lasnier, G. Hannink, G. J. Verkerke, and N. Verdonschot. Experimental assessment of a new direct fixation implant for artificial limbs. J. Mech. Behav. Biomed. Mater. 21:77-85, 2013.

${ }^{39}$ Tomaszewski, P. K., M. van Diest, S. K. Bulstra, N. Verdonschot, and G. J. Verkerke. Numerical analysis of an osseointegrated prosthesis fixation with reduced bone failure risk and periprosthetic bone loss. J. Biomech. 45:18751880, 2012.

${ }^{40}$ Turner, C. H., I. Owan, and Y. Takano. Mechanotransduction in bone: role of strain rate. Am. J. Physiol. Endocrinol. Metab. 269:E438-E442, 1995.

${ }^{41}$ Viceconti, M., L. Cristofolini, M. Baleani, and A. Toni. Pre-clinical validation of a new partially cemented femoral prosthesis by synergetic use of numerical and experimental methods. J. Biomech. 34:723-731, 2001.

${ }^{42}$ Viceconti, M., S. Olsen, L.-P. Nolte, and K. Burton. Extracting clinically relevant data from finite element simulations. Clin. Biomech. 20:451-454, 2005.

${ }^{43}$ Weinans, H., R. Huiskes, and H. J. Grootenboer. The behavior of adaptive bone-remodeling simulation models. J. Biomech. 25:1425-1441, 1992.

${ }^{44} \mathrm{Xu}, \mathrm{W}$., and $\mathrm{K}$. Robinson. X-Ray image review of the bone remodeling around an osseointegrated trans-femoral implant and a finite element simulation case study. Ann. Biomed. Eng. 36:435-443, 2008.

${ }^{45}$ Zanetti, E. Z., A. Aldieri, M. Terzini, M. Calì, G. Franceschini, and C. Bignardi. Additively manufactured custom load-bearing implantable devices: grounds for caution. AMJ 10(8):694-700, 2017.

Publisher's Note Springer Nature remains neutral with regard to jurisdictional claims in published maps and institutional affiliations. 